Revisión

\title{
Revisión de la Literatura Sobre el Problema de Programación de "Flow Shop" Híbrido con Máquinas Paralelas no Relacionadas
} Literature Review on the Hybrid Flow Shop Scheduling Problem with Unrelated Parallel Machines

Eliana Peña Tibaduiza*1, Edwin Alberto Garavito Hernandez ${ }^{1}$, Luis Pérez Figueredo $^{1}$, Eslendis Moratto Chimenty ${ }^{1}$

${ }^{1}$ Universidad Industrial de Santander. Colombia

*Correo electrónico: elmapena@uis.edu.co

Recibido: 05-04-2016. Modificado: 16-08-2016. Aceptado: 10-10-2016

\section{Resumen}

Contexto: Se ha observado que el problema de flow shop híbrido con máquinas paralelas no relacionadas presenta escasos reportes en el ámbito académico, en comparación con el flow shop híbrido que considera procesadores idénticos, razón por la cual son poco conocidos los diferentes enfoques y aplicaciones que este problema puede tener.

Método: Se realizó una revisión de la literatura del estado del arte del problema de programación de trabajos en un flow shop híbrido mediante la recopilación y análisis de artículos académicos en diversas bases de datos científicas. Con este propósito se realizó una búsqueda a partir de palabras claves que definen el problema, revisando si evidencian en la descripción del problema la existencia de máquinas paralelas no relacionadas; como resultado se obtienen 50 artículos seleccionados para el presente estudio.

Resultados: Se realizó una clasificación del problema de acuerdo a las características del sistema productivo y se presentan los métodos de solución, restricciones y funciones objetivo comúnmente utilizadas.

Conclusiones: Se observa una tendencia creciente en los estudios del flow shop con múltiples etapas, pero pocos son los estudios basados en casos de la industria.

Palabras clave: "Flow shop" híbrido, máquinas paralelas no relacionadas, programación, revisión de la literatura.

Idioma: Español

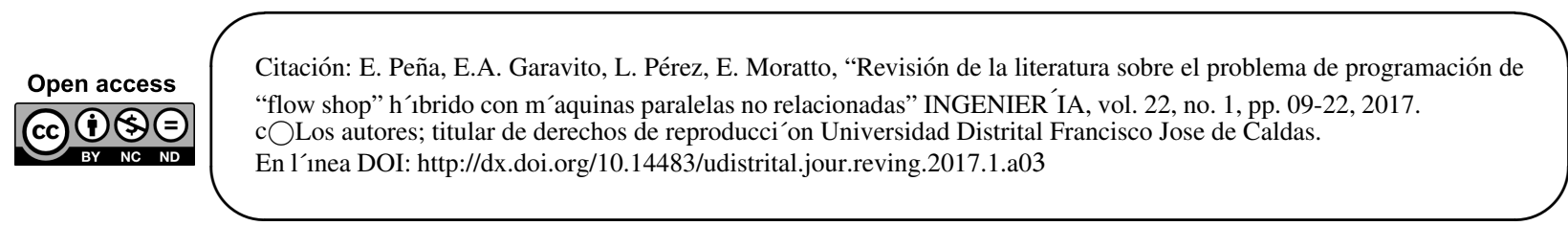




\begin{abstract}
Context: The flow shop hybrid problem with unrelated parallel machines has been less studied in the academia compared to the flow shop hybrid with identical processors. For this reason, there are few reports about the kind of application of this problem in industries.

Method: A literature review of the state of the art on flow-shop scheduling problem was conducted by collecting and analyzing academic papers on several scientific databases. For this aim, a search query was constructed using keywords defining the problem and checking the inclusion of unrelated parallel machines in such definition; as a result, 50 papers were finally selected for this study.
\end{abstract}

Results: A classification of the problem according to the characteristics of the production system was performed, also solution methods, constraints and objective functions commonly used are presented.

Conclusions: An increasing trend is observed in studies of flow shop with multiple stages, but few are based on industry case-studies.

Keywords: Hybrid flow shop, unrelated parallel machine, literature review, scheduling.

\title{
1. Introducción
}

En un sistema productivo tipo flow shop un conjunto de tareas debe seguir un flujo unidireccional para ser procesado a través de múltiples estaciones, donde cada estación consta de una sola máquina. En algunas fábricas, la necesidad de aumentar o equilibrar la capacidad ha llevado a la adquisición de nuevos procesadores en algunas etapas [1], los cuales pueden coexistir con los ya disponibles.

Normalmente las máquinas adquiridas tienen características diferentes de las existentes, pueden llegar a ser más rápidas, tienen un menor tiempo de alistamiento, pueden procesar más trabajos, entre otras, y son conocidas en la literatura como máquinas no relacionadas. Este tipo de sistemas es frecuente en industrias como las de fabricación de baldosas cerámicas, automotriz, metalmecánicas, de fabricación de televisores con circuito impreso a bordo y suele ser denominado flow shop híbrido Hybrid Flow Shop (HFS, por sus siglas en inglés) o flow shop flexible, Flexible Flow Shop (FFS, por sus siglas en inglés).

Tal problema ha sido del interés de numerosos investigadores desde que aparecieran los primeros trabajos en 1970, dada su complejidad y aplicación práctica. Se han planteado diferentes métodos tanto heurísticos como exactos para hallar una solución factible considerando diferentes restricciones. Tradicionalmente, los trabajos que se han realizado sobre flow shop híbrido han estudiado la restricción de máquinas paralelas idénticas, caso que no es muy común en las industrias manufactureras.

Es por esto que este trabajo presenta una revisión de las investigaciones desarrolladas considerando máquinas paralelas no relacionadas, con el propósito de complementar las revisiones realizadas por Ribas et al. [1], Morais et al. [2], Wenpeng Wang [3], Ruiz y Vázquez [4], en las cuales un porcentaje muy bajo de los estudios consultados corresponden a esta área. En el presente trabajo 
se hace una revisión del problema determinístico, siendo este en el que se encuentran la mayor cantidad de reportes.

El contenido de este artículo se organiza como se describe a continuación: en la sección dos se presenta la definición del problema. Posteriormente, el apartado tres describe el protocolo de investigación seguido por la presentación de los resultados obtenidos (sección cuatro). Por último, se presentan las conclusiones en el apartado cinco.

\section{Descripción del problema HFS}

El problema de flow shop híbrido, Hybrid Flow Shop (HFS, por sus siglas en inglés), tiene como objetivo determinar de forma adecuada la secuencia y asignación de un conjunto de tareas a un conjunto de $m$ máquinas para lograr optimizar una función en particular; dicha función considerada por lo general es el makespan o la cantidad de trabajos tardíos. En este problema se considera que los trabajos tienen el mismo orden de procesamiento y el flujo del proceso es unidireccional.

El HFS como se ha venido trabajando, no contempla aspectos importantes de la producción en las industrias reales, debido a que los estudios que generalmente se hacen no consideran parámetros y supuestos presentes en la realidad como la variación en los tiempos de alistamiento y de operación a partir de un enfoque estocástico, al igual que se excluye en la mayoría de los casos la posibilidad de un tratamiento dinámico del problema considerando las variaciones de los parámetros en periodos diferentes. De igual manera sucede con la existencia de máquinas paralelas no relacionadas. Es por esto que las revisiones que se han realizado sobre este tema son pocas y no agrupan todas las posibles variantes que puede tener este problema, hecho que conlleva a la no claridad acerca de su definición, es decir, no hay unanimidad de los conceptos, pues mientras unos autores consideran flow shop híbrido a aquel que tiene máquinas paralelas idénticas y flow shop flexible al de máquinas paralelas no relacionadas, distintos autores dicen lo contrario. Teniendo en cuenta lo dicho anteriormente se presenta una clasificación del problema de flow shop híbrido con máquinas paralelas no relacionadas, de acuerdo a lo reportado en la literatura por investigadores como Lin y Chen [5], Ewa Figielska [6], entre otros, considerando además el enfoque del problema como determinístico y estático.

\section{Protocolo de investigación}

El proceso de investigación inició con una revisión acerca del problema de flow shop híbrido con máquinas paralelas no relacionadas, teniendo en cuenta lo expuesto por Pautasso [7], quien propone diez reglas para escribir una revisión de la literatura. Asimismo, se plantean una serie de preguntas a resolver, similar a lo planteado por [8], que permiten estructurar el presente trabajo; dichas cuestiones están enfocadas a identificar cuáles son las restricciones, medidas de desempeño y métodos de solución más utilizados.

Se definió una ventana de tiempo entre los años 1995 y 2015, a su vez se construyó una ecuación de búsqueda identificando como palabras claves: "hybrid flow shop", "flexible flow shop", 
"unrelated parallel machine", "scheduling". La ecuación de búsqueda se introdujo en las siguientes bases de datos: Springer Link, Elsevier, Proquest, Scopus, Web of Science e IEEE/IEE Electronic Library. Los resultados de esta consulta se resumen en la Tabla I, aclarando que en los resultados se encuentran artículos repetidos, para finalmente obtener 543 documentos diferentes.

En la selección de los documentos se consideró el hecho que no existe consenso en el medio académico sobre el nombre que recibe el problema, es decir, algunos autores consideran como flow shop híbrido aquel sistema que tiene máquinas paralelas idénticas y flow shop flexible el que presenta procesadores no relacionados, fue necesario revisar que los documentos encontrados evidenciaran en la descripción del problema la existencia de máquinas paralelas no relacionadas. Igualmente, se aplica otro filtro verificando criterios de inclusión considerando que el documento corresponda a un artículo completo; de igual manera, se estable- ce que el contenido del documento debe presentar la descripción del modelo matemático, incluyendo la definición de la función objetivo, las restricciones y parámetros, además del método o métodos de solución planteados por los autores.

Tabla I. Resultados de la consulta inicial.

\begin{tabular}{lc}
\multicolumn{1}{c}{ Base de Datos } & Cantidad \\
\hline Science Direct - Elsevier & 252 \\
\hline Web of Science & 194 \\
\hline SCOPUS & 361 \\
\hline Proquest & 280 \\
\hline Springer Link & 205 \\
\hline IEEE & 19 \\
\hline
\end{tabular}

A partir de este filtro se depura la cantidad de artículos con los que se realiza la extracción de datos para continuar con la última etapa, en la cual se analizan 50 artículos, que corresponden a los que cumplieron con los criterios de inclusión. Posteriormente se realizó una síntesis sobre cada uno de los aspectos del problema buscando identificar diferentes enfoques y tendencias (restricciones, métodos de solución, publicaciones por año, etc.).

Esta revisión se enfoca en un tópico de investigación preciso, con el ánimo de brindar aspectos de interés para los investigadores de esta área, por tal razón puede considerarse una "mini-revisión", según lo describe Velásquez [9].

\section{Resultados}

En esta sección se muestran los resultados en cuanto a estadísticas descriptivas de la investigación realizada; es importante resaltar que al revisar los artículos seleccionados se encuentra que el $78,9 \%$ de ellos utilizan el término "híbrido" para referirse al problema en estudio, por esta razón se utilizará dicho término para la descripción de los hallazgos; es así como se observa cuáles son las tendencias de estudio del problema de flow shop híbrido con procesadores no relacionados.

\subsection{Análisis de resultados}

La figura 1 muestra el número de artículos publicados por año. Al analizar esta figura, se puede observar que existe una tendencia de crecimiento en las investigaciones del problema de flow shop híbrido con máquinas paralelas no relacionadas. 
Si bien son varios los autores que han publicado sobre este problema, en los artículos encontrados para esta revisión, se observó que Ewa Figielska [6], [10]-[12] [6], [10]-[12] y Jitti Jungwattanakit [13]-[15] son los investigadores que más han incursionado en su estudio, cada uno considerando diversas restricciones: buffers de capacidad limitada y existencias de recursos no renovables por parte de Figielska y tiempos de alistamiento dependientes de la secuencia, tiempos de alistamientos dependientes de la máquina, elegibilidad de máquinas y tiempos de liberación por Jungwattanakit.

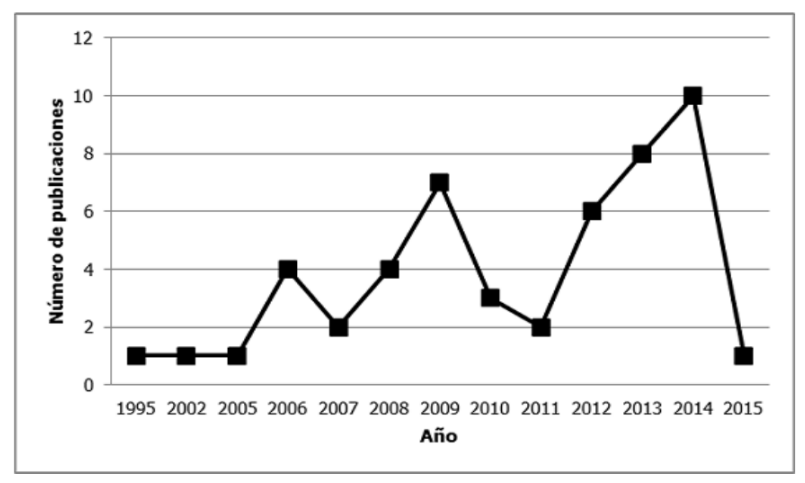

Figura 1. Número de publicaciones por año: detalle del número de artículos por cada año desde 1995 hasta 2015 que fueron encontrados en bases de datos científicas.

\subsection{Clasificación del problema}

Con base en lo expuesto por Linn y Zahng [15], el HFS puede ser clasificado en términos de la complejidad del proceso en tres categorías descritas a continuación.

\subsubsection{HFS con dos etapas}

Considera la existencia de solo dos etapas de procesamiento bajo diferentes configuraciones tecnológicas. En esta categoría se pueden describir tres casos: el primero de ellos en el cual hay una sola máquina en la primera etapa y $m$ máquinas paralelas no relacionadas en la segunda etapa; esta variación ha sido estudiada por Uetake et al. [17], quienes consideran el procesamiento de trabajos por lotes o familias y posteriormente por Almeder y Hartl [18], quienes lo estudian a través de un caso de aplicación en la industria metalúrgica.

Figielska [6] y Low et al. [19], presentan la segunda variante de este problema: $m$ máquinas no relacionadas en la primera etapa y una máquina en la segunda. La primera considerando restricciones como preferencia de trabajo y el segundo teniendo en cuenta las restricciones de precedencia y elegibilidad de máquinas. Asimismo, la tercera y última variación: $m$ máquinas no relacionadas en ambas etapas es investigada por Figielska [12] y Rabiee et al. [20].

\subsubsection{HFS con múltiples etapas}

Se han estudiado sistemas productivos que consisten de tres etapas de procesamiento en la que al menos una etapa posee múltiples máquinas paralelas no relacionadas. Sobre esta clasificación Yaurima et al., realizan dos investigaciones: en 2008 [21] en industrias de fabricación de televisores con circuito impreso a bordo y luego en 2009 [20] incluyendo las zonas de almacenamiento intermedias o buffers con capacidad limitada.

Posteriormente Li et al. [23] abordan el problema considerando restricciones de grupo en la segunda etapa y proponen diez algoritmos, nueve de ellos basados en el algoritmo Shop Partitioning $(S P)$ o enfoque de descomposición orientado a las etapas y uno basado en el algoritmo Three Stage 
(TS) es decir, el enfoque holístico que considera el problema de programación.

De igual forma se ha investigado sobres trabajos que deben ser procesados en más de tres etapas. Dicho problema ha sido estudiado por Nagano y Mocellin [24] cuando lo consideran como dos flow shop tradicionales funcionando en paralelo. Posteriormente, el problema fue abordado por Chinyao Low [25], quien propone una heurística basada en recocido simulado para su solución.

Así como el HFS de dos etapas, el HFS de múltiples etapas presenta variaciones: la primera de ellas es el flow shop híbrido flexible, en el que los trabajos pueden omitir algunas de las etapas de procesamiento. Esta variante ha sido estudiada por Ruiz et al. [26], Zandieh et al. [27], Bozogirad y Logendran [28], Attar et al. [29], Siqueira et al. [30], Li et al. [31] y Arango et al. [32].

La segunda variante de esta clasificación es el flow shop híbrido cíclico o flow shop híbrido reentrante, considerada por Kim y Lee [33] cuando evalúan la tardanza total y el makespan y Soltani y Karimi [34], que consideran el problema sujeto a restricciones de elegibilidad de máquinas.

\subsection{Restricciones comunes}

Como se había mencionado antes, el HFS es un problema que permite modelar sistemas productivos reales si se tienen en cuenta limitaciones que se presentan a lo largo del sistema productivo, estas limitaciones generalmente tienen que ver con las tareas o grupos de tareas a ser procesadas, las máquinas y las políticas de operación. A continuación, se describen las restricciones comúnmente encontradas en la literatura:

Tiempos de alistamiento: Jungwattanakit et al. [13] en 2006 clasifican estos tiempos en dos tipos: dependientes de la máquina y dependientes de la secuencia. Un tiempo de configuración de un trabajo es dependiente de la máquina, si depende de la máquina a la cual ha sido asignado y solo ocurre con el primer trabajo asignado al procesador. Un tiempo de preparación dependiente de la secuencia es considerado entre trabajos sucesivos, es decir depende del trabajo asignado anteriormente en la máquina.

Elegibilidad de máquinas: no todas las máquinas existentes en cada estación son capaces de procesar todos los trabajos [35].

Existencia de Buffers: zonas de almacenamiento entre estaciones que pueden o no tener capacidad limitada.

Tiempos de transporte: consideran los tiempos de transferencia del producto entre estaciones.

Tiempos de liberación de máquinas: ninguna máquina puede procesar una nueva tarea hasta que no haya sido liberada. La liberación ocurre luego de transcurrir un intervalo de tiempo que incluye el procesamiento y el bloqueo, en caso que este se presente.

No espera: a los trabajos no les es permitido esperar entre dos máquinas sucesivas [35]. 
Tabla II. Restricciones consideradas en las publicaciones

\begin{tabular}{|c|c|}
\hline Restricciones & Publicación donde ha sido considerada \\
\hline Tiempos de alistamiento & $\begin{array}{l}\text { Uetake } \text { et al. [17], Low [25], Ruiz y Maroto [36], Jitti } \text { et al. [13]-[15], Gómez [37], Ruíz } \\
\text { et al. [26], Yaurima } \text { et al. [21], [22],Rashidi et al. [38], Defersha y Chen [39], Zandieh } \text { et } \\
\text { al. [27], Gómez Gasquet [40], Lugo y Teixeira [41], Bozorgirad y Logendran [28], Lugo } \\
\text { et al. [42], Attar } \text { et al. [29], Siqueira } \text { et al. [30], Dudas } \text { et al. [49], [54] }\end{array}$ \\
\hline Elegibilidad de máquinas & $\begin{array}{l}\text { Ruíz y Maroto [36], Ruíz et al. [26], Yaurima et al. [20], [21], Defersha y Chen [39], } \\
\text { Lugo y Teixeira [41], Bozorgirad y Logendran [28], Lugo et al. [42], Siqueira et al. [30], } \\
\text { Soltani y Karimi [34] }\end{array}$ \\
\hline Existencia de buffers & $\begin{array}{l}\text { Jenabi } \text { et al. [43], Figielska [6], [10], [11], Yaurima et al. [21], [22], Xu et al. [44], Al- } \\
\text { meder y Hartl [18], Lugo et al. [42], Soltani y Karimi [43], Li et al. [32], Li y Pan [45] }\end{array}$ \\
\hline Tiempos de liberación de máquinas & $\begin{array}{l}\text { Jitti et al. [14], [15], Ruíz et al. [26], Scholz-Reiter } \text { et al. [46], Zandieh } \text { et al. [27], } \\
\text { Bozorgirad y Logendran [28], Lugo et al. [42], Siqueira } \text { et al. [30], Li et al. [23] }\end{array}$ \\
\hline No espera & Rabiee et al. [20] \\
\hline De precedencia & Ruiz et al. [26], \\
\hline De preferencia & Parra y Mejía [46], Figielska [6], [10]-[12] \\
\hline
\end{tabular}

De precedencia: ciertos trabajos deben ser completados antes que otros puedan empezar su procesamiento [35].

De preferencia: se refiere a que el procesamiento de un trabajo en una máquina puede ser interrumpido e incluso posteriormente terminado en otra máquina dada una prioridad asignada a cada trabajo [35].

En la Tabla II, se presentan las restricciones que fueron consideradas en la mayoría de las publicaciones, encontrando que los tiempos de alistamiento dependientes de la secuencia y dependientes de la máquina, elegibilidad de máquinas, existencia de buffers y tiempos de liberación de máquinas son las restricciones más utilizadas.

\subsection{Medidas de desempeño}

Una de las medidas de desempeño utilizadas en mayor medida es minimizar el makespan, considerada por autores como: Jitti et al. [14], Yaurima et al. [22], Ruiz y Maroto [36], GómezGasquet [40] y Defersha y Chen [39].

En la gran mayoría de los casos, los autores se inclinan por una única función objetivo, pero exis-

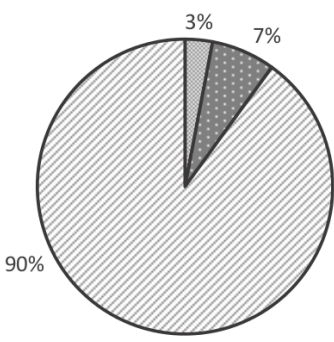

口Tiempo de ciclo $\boldsymbol{\square}$ Tardanza total $\boldsymbol{\square}$ Makespan

Figura 2. Medidas de desempeño en una sola función objetivo: muestra el porcentaje de uso de cada una de las medidas de desempeño que fueron utilizadas cuando se evaluó una función.

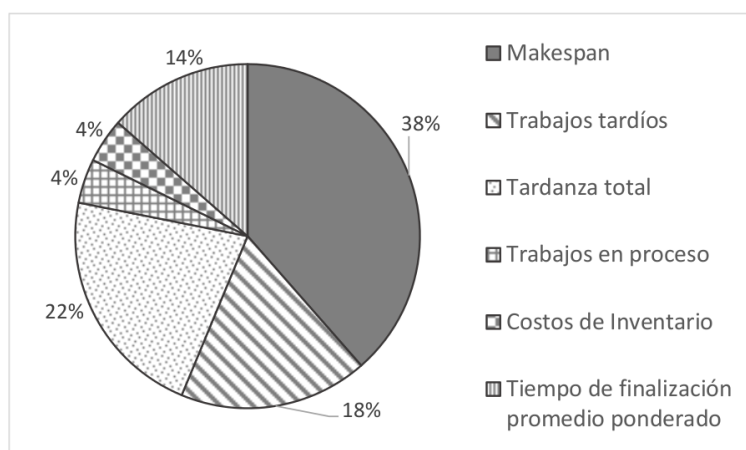

Figura 3. Medidas de desempeño en problemas biobjetivo: detalla en que porcentaje fueron utilizadas las medidas de desempeño cuando se evaluaban dos funciones objetivo. 
ten aquellos que consideran dos y más medidas de desempeño al tiempo. Tal es el caso de Dai et al. [48], quienes buscan minimizar el makespan y el consumo energético de las máquinas, Bozorgirad y Logendran [28] plantean como objetivo minimizar el tiempo de finalización total ponderado y la tardanza total ponderada.

Las figuras 2, 3 y 4, muestran cuáles son las medidas de desempeño adoptadas por los autores cuando evalúan una, dos y tres funciones objetivas respectivamente. En el caso de más de dos de funciones se encuentran trabajos como el de Dudas [48], quienes plantean minimizar el producto en proceso, Work in Process (WIP, por sus siglas en inglés) promedio, la tardanza total y el makespan; también Almeder y Hartl [18] presentan un caso de aplicación en la industria metalúrgica de un flow shop de dos etapas con existencia de un buffer limitado entre ellas; se plantearon cuatro objetivos a cumplir: utilización de las máquinas; evitar bloqueos en la máquina de la etapa uno; tiempo de finalización

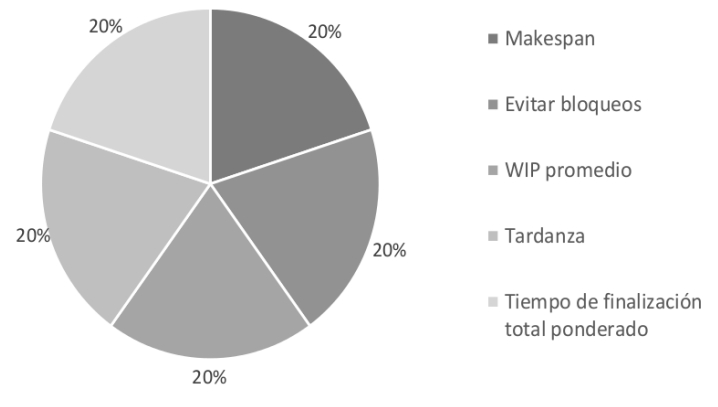

Figura 4. Medidas de desempeño en problemas multiobjetivo: muestra que cada una de las medidas de desempeño fueron evaluadas por igual en los problemas que consideraban más de dos funciones objetivos. promedio esperado.

\subsection{Métodos de solución}

Al resolver el problema de programación en un sistema productivo flow shop híbrido con máquinas paralelas no relacionadas muchos han sido los métodos empleados. De Fatima Morais et al. [2] clasifican estos métodos de solución en dos tipos (figura 5): métodos óptimos o exactos y métodos aproximados. Es de aclarar que los modelos matemáticos hacen referencia al planteamiento del problema como un modelo de programación lineal.

Ruiz y Maroto [49] definen que las heurísticas se pueden dividir en heurísticas constructivas o en heurísticas de mejora. Las heurísticas constructivas generan el paso a paso de la solución al problema, son deterministas y se basan en la mejor elección en cada iteración [51], mientras que las heurísticas de mejora toman una solución encontrada inicialmente por algún procedimiento iterativo y la modifican buscando una solución mejor de acuerdo a los objetivos definidos [2].

Finalmente, Chicano [52] en su tesis doctoral define las metaheurísticas como la combinación de diferentes métodos heurísticos a un nivel más alto para conseguir una exploración del espacio de búsqueda de forma eficiente y efectiva; a su vez clasifica las metaheurísticas en dos tipos: basadas en trayectoria y basadas en población.

Las metaheurísticas basadas en trayectoria parten de una solución y, mediante la exploración del vecindario, van actualizando la solución inicial formando una trayectoria. Aquí pertenecen al recocido simulado. Simulated Annealing (SA, por sus siglas en inglés); búsqueda tabú, Tabu Search (TS, por sus siglas en inglés); procedimiento de búsqueda miope aleatorizado y adaptativo, Greddy 


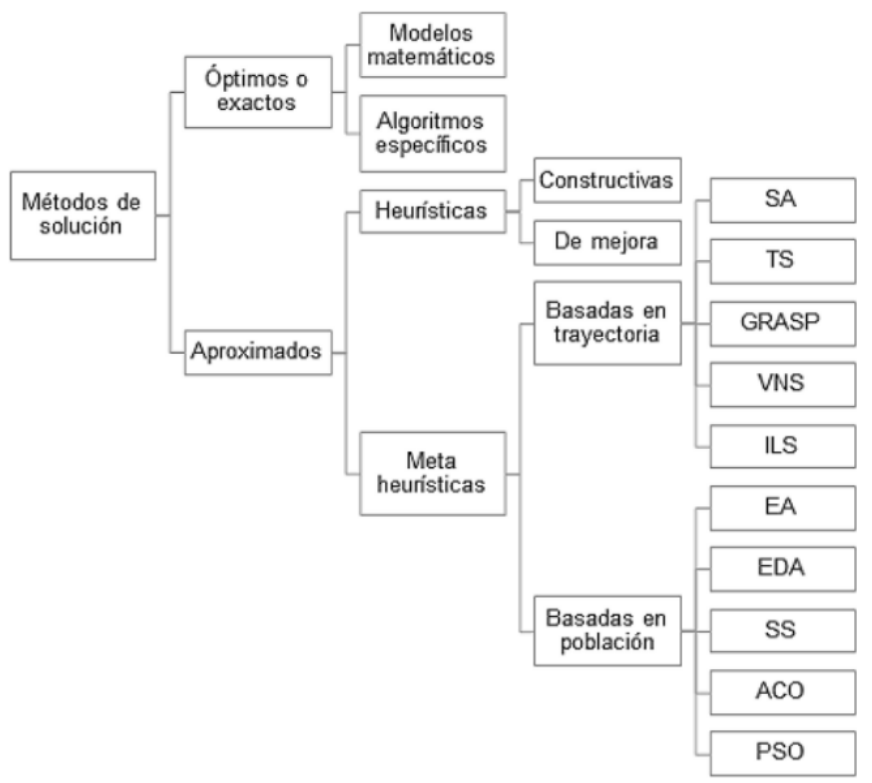

Figura 5. Clasificación de los métodos de solución. Presenta una adaptación de la clasificación de los métodos de solución realizada por O. Suarez [50]

Randomized Adaptive Search (GRASP, por sus siglas en inglés); búsqueda con vecindario variable, Variable Neighborhood Search (VNS, por sus siglas en inglés); búsqueda local iterada, Iterated Local Search (ILS, por sus siglas en inglés).

Por su parte, las metaheurísticas basadas en población se caracterizan por trabajar un conjunto de soluciones (llamado población) en cada iteración. En este grupo se encuentran: algoritmos evolutivos, Evolutionary Algorithm (EA, por sus siglas en inglés); algoritmos de estimación de la distribución, Estimation of Distribution Algorithm (EDA, por sus siglas en inglés); búsqueda dispersa, Scatter Search (SS, por sus siglas en inglés); optimización basada en colonia de hormi-

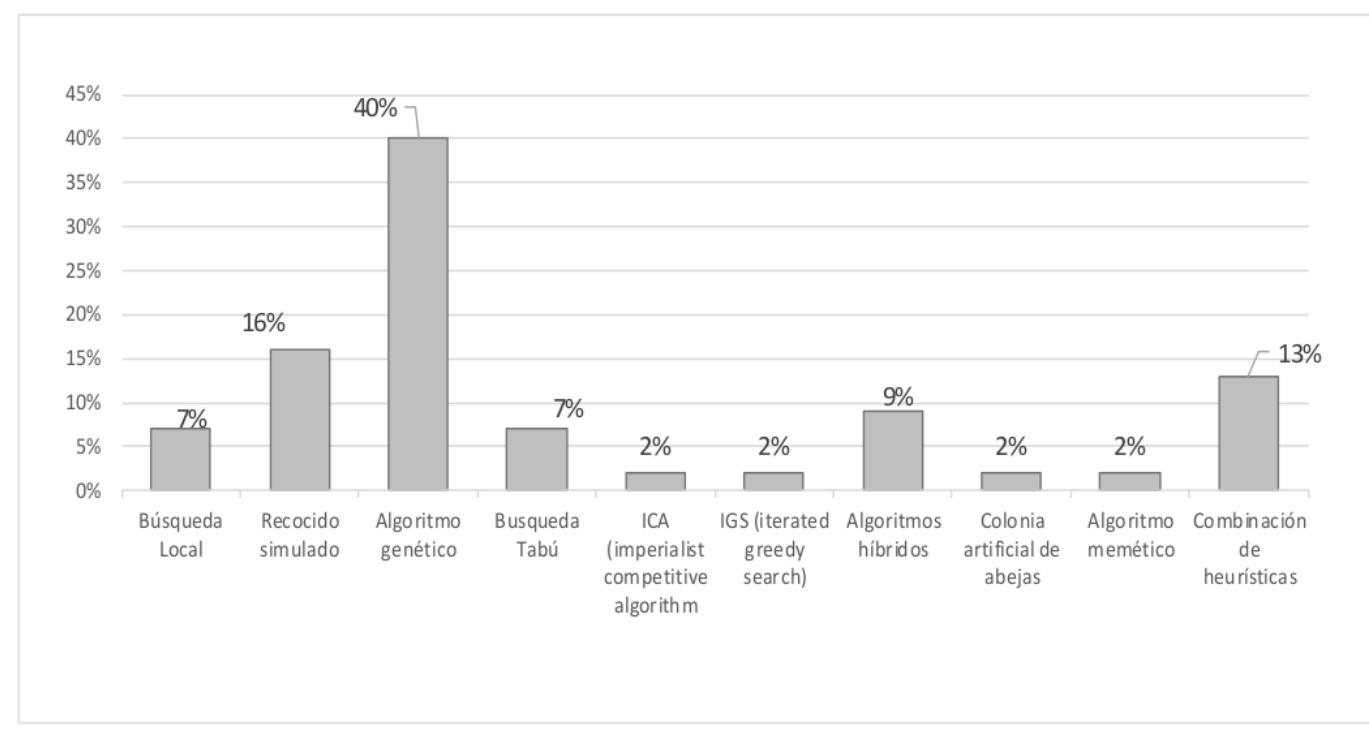

Figura 6. Métodos de solución utilizados: enseña el porcentaje de un total de 50 documentos, en que fue utilizado cada método de solución para resolver el problema dentro de las investigaciones realizadas. 
gas, Ant Colony Optimization (ACO, por sus siglas en inglés); optimización basada en cúmulos de partículas, Particle Swarm Optimization (PSO, por sus siglas en inglés).

De acuerdo con el análisis realizado de las publicaciones encontradas (figura 6), se observó que el método utilizado en mayor medida es el algoritmo genético, considerado por autores como: Figielska [10], Zandieh et al. [27], Jun y Park [53], Lugo et al. [41], entre otros. Por el contrario, los menos utilizados son: el algoritmo memético, la colonia artificial de abejas, IGS e ICA, adoptados por Xu et al. [44], Li y Pan [45], Siqueira et al. [30] y Rabiee et al. [20], respectivamente.

\section{Conclusiones}

En este artículo se presenta una revisión de 50 publicaciones sobre el problema de flow shop híbrido con máquinas paralelas no relacionadas desde 1995 hasta 2015, las cuales han sido clasificadas considerando la complejidad del proceso productivo. Otros aspectos considerados son los métodos de solución planteados, las restricciones del sistema productivo y las medidas de desempeño.

A partir de dicha revisión es posible concluir que uno de los métodos de solución comúnmente utilizados son los algoritmos genéticos y el algoritmo de recocido simulado. Por su parte, la implementación de algoritmos híbridos y metaheurísticas basadas en poblaciones como lo son los algoritmos memético o la optimización basada en colonia de hormigas (ACO) ha sido muy baja dentro de los estudios realizados, en comparación con los Algoritmos Genéticos que han sido la metaheurística más utilizada.

El análisis de las investigaciones realizadas en los últimos años muestra una tendencia creciente en los estudios del problema de Flow shop híbrido con múltiples etapas y sus variantes con un solo objetivo o múltiples objetivos, sin embargo, son pocos los casos de aplicación en las diferentes industrias los que han sido registrados en la literatura. Se han encontrado reportes de casos de fabricación de baldosas cerámicas, automotriz, metalmecánicas y de fabricación de televisores con circuito impreso a bordo. Es por esto que se evidencia un trabajo por fortalecer en llevar soluciones planteadas a las organizaciones.

Con relación a las medidas de desempeño, la minimización del makespan continúa siendo una de las más comunes junto con la minimización de la tardanza total y del tiempo de finalización total ponderado. El porcentaje de los trabajos que se enfocan en objetivos como la utilización eficiente de los diferentes insumos requeridos para la producción como el consumo energético o la disponibilidad de trabajadores es bajo.

En cuanto a las restricciones consideradas, son los tiempos de alistamiento dependientes de la secuencia y la existencia de buffers con capacidad limitada las más utilizadas. Por el contrario, restricciones como el fraccionamiento de trabajos en lotes de procesamiento, tiempos de alistamiento dependientes de la máquina y tiempos de transporte entre etapas han sido poco estudiadas.

Otro aspecto a fortalecer son las instancias disponibles en la literatura para realizar validaciones de resultados de nuevas metaheurísticas híbridas que se han venido desarrollando, pues no se en- 
cuentran instancias con las características específicas, como por ejemplo para el caso del flow shop híbrido con máquinas paralelas no relacionadas con tiempos de alistamiento dependientes de la secuencia, para que así los autores puedan comparar los resultados y poder establecer la eficiencia de los algoritmos propuestos.

Dado que no se ha encontrado un gran número de artículos científicos abordando diferentes variantes al problema del flow shop híbrido con máquinas paralelas no relacionadas, tanto en cantidad de etapas como en sus restricciones y objetivos, se sugiere considerar restricciones como capacidad y recursos limitados, tiempos de transferencia entre etapas de procesamiento, zonas de almacenamiento entre etapas de procesamiento, entre otras, de forma que se puedan comparar los resultados y ampliar el conocimiento desarrollado hasta el momento. Asimismo, revisando trabajos previamente desarrollados y llegar a proponer modificaciones, como por ejemplo en algoritmos genéticos los operadores de cruce y mutación.

De igual forma, el problema del flow shop híbrido estocástico es una línea de investigación que aún no ha sido ampliamente estudiada y que puede traer consigo futuras investigaciones que lleven a solucionar problemas más cercanos a la realidad de las industrias, considerando la variabilidad de las operaciones, alistamientos, transportes y demás actividades inmersas en los procesos de fabricación. En trabajos como el de Ding et al. [54] se declara un interés en acercarse más a las situaciones reales de las industrias, incluyendo en su estudio la variabilidad en los tiempos de preparación y terminación.

\section{Referencias}

[1] Imma Ribas, Rainer Leisten, y Jose. M. Framiñan, "Review and classification of hybrid flow shop scheduling problems from a production system and a solutions procedure perspective". Comput. Oper. Res., vol. 37, no. 8, 2010, pp. 1439-1454. 10

[2] Marcia de Fatima Morais, Thays. J. Perassoli , Leandro dos Santos, Rony Peterson, y Paulo Paraíso, "Multicriteria Hybrid Flow Shop Scheduling Problem: Literature Review, Analysis, and Future Research". Indep. J. Manag. Prod., vol. 5, no. 4, pp. 2014, 1004-1031. 10, 16

[3] Wenpeng Wang, "Review on hybrid flow shop scheduling”. Int. Conf. Inf. Technol. Comput. Eng. Manag. Sci. ICM 2011, vol. 4, 2011 pp. 7-10. 2011. 10

[4] Ruben Ruiz y Jose Vázquez, “The hybrid flow shop scheduling problem”. Eur. J. Oper. Res., vol. 205, no. 1,2010, pp. $1-18.10$

[5] James Lin y Chien Chen, "Simulation optimization approach for hybrid flow shop scheduling problem in semiconductor back-end manufacturing”. Simul. Model. Pract. Theory, vol. 51, 2015, pp. 100-114.11

[6] Ewa Figielska, "A new heuristic for scheduling the two-stage flowshop with additional resources". Comput. Ind. Eng., vol. 54, no. 4, 2008pp. 750-763. 11, 13, 15

[7] Marco Pautasso, “Ten simple rules for writing a literature review”. PLoS Comput. Biol., vol. 9, no. 7, 2013, pp. $1-4.11$

[8] Cesar Pineda y Cesar López, Üna revisión al estado del arte de la integración de toma de decisiones en la red logística", Ingeniería, vol. 18, Num. 1, pp 118-148. 11

[9] Juan D. Velásquez, Üna Guía Corta para Escribir Revisiones Sistemáticas de Literatura Parte 1”. Dyna, 81. 187, 2014, pp9-10. 12

[10] Ewa Figielska, "A genetic algorithm and a simulated annealing algorithm combined with column generation technique for solving the problem of scheduling in the hybrid flowshop with additional resources". Comput. Ind. Eng., vol. 56, no. 1, 2009, pp. 142-151. 13, 15, 18 
[11] Ewa Figielska, "Heuristic algorithms for preemptive scheduling in a two-stage hybrid flowshop with additional renewable resources at each stage". Comput. Ind. Eng., vol. 59, no. 4, 2010, pp. 509-519. 13, 15

[12] Ewa Figielska, "A heuristic for scheduling in a two-stage hybrid flowshop with renewable resources shared among the stages". Eur. J. Oper. Res., vol. 236, no. 2, 2014, pp. 433-444. 13, 15

[13] Jitti Jungwattanakit, Manop Reodecha, Paveena. Chaovalitwongse y Frank. Werner, "Constructive and simulated annealing heuristics for hybrid flow shops with unrelated parallel machines". in Proceedings of the 3rd OR-CRN Operations Research Conference, Bangkok, Thailand, 2006, pp. 110-121. 13, 14, 15

[14] Jitti Jungwattanakit, Manop Reodecha, Paveena Chaovalitwongse, y Frank Werner, "Algorithms for flexible flow shop problems with unrelated parallel machines, setup times, and dual criteria". Int. J. Adv. Manuf. Technol., vol. 37, no. 3-4, 2008, pp. 354-370. 13, 15

[15] Jitti Jungwattanakit, Manop Reodecha, P Chaovalitwongse, y Frank Werner, "A comparison of scheduling algorithms for flexible flow shop problems with unrelated parallel machines, setup times, and dual criteria". Comput. Oper. Res., vol. 36, no. 2, 2009, pp. 358-378. 13, 15

[16] Richard Linn y Wei Zhang, "Hybrid flow shop scheduling: a survey". Comput. Ind. Eng., vol. 37, no. 1, 1999, pp. 57-61.

[17] Toshifumi Uetake, Hitoshi Tsubone, y Masaaki Ohba, "A production scheduling system in a hybrid flow shop". Int. J. Prod. Econ., vol. 41, no. 1-3, 1995, pp. 395-398.13, 15

[18] Christian Almeder y Richard Hartl, "A metaheuristic optimization approach for a real-world stochastic flexible flow shop problem with limited buffer". Int. J. Prod. Econ., vol. 145, no. 1, 2013, pp. 88-95.13, 15, 16

[19] Chinyao Low, Chou Hsu, y Chwen Su, "A two-stage hybrid flowshop scheduling problem with a function constraint and unrelated alternative machines". Comput. Oper. Res., vol. 35, no. 3, 2008, pp. 845-853. 13

[20] M. Rabiee, R. Sadeghi Rad, M. Mazinani, y R. Shafaei, "An intelligent hybrid meta-heuristic for solving a case of no-wait two-stage flexible flow shop scheduling problem with unrelated parallel machines". Int. J. Adv. Manuf. Technol., vol. 71, no. 5-8, 2014, pp. 1229-1245.13, 15, 18

[21] Victor Yaurima, Larisa Burtseva, y Andrei Tchernykh, "Hybrid Flowshop with Unrelated Machines, Sequence Dependent Setup Time and Availability Constraints : An Enhanced Crossover Operator for a Genetic Algorithm". Springer verlag Berlin Heidelberg, 2008, pp. 608-617.13, 15

[22] Victor Yaurima, Larisa Burtseva, y Andrei Tchernykh, "Hybrid flowshop with unrelated machines, sequencedependent setup time, availability constraints and limited buffers". Comput. Ind. Eng., vol. 56, no. 4, 2009, pp. 1452-1463. 15

[23] Zhan Tao Li, Jianjun Liu, Qing Chen, Ning Mao, and Xiaoming Wang, "Approximation algorithms for the threestage flexible flow shop problem with mid group constraint". Expert Syst. Appl., vol. 42, no. 7, 2015, pp. 3571-3584. 13,15

[24] Marcelo Seido Nagano, "Flowshops Paralelos Com Processadores Não-Relacionados". XXII Encontro Nacional de Engenharia de Produção, 2002, pp. 1-8.14

[25] Chinyao Low, "Simulated annealing heuristic for flow shop scheduling problems with unrelated parallel machines". Comput. Oper. Res., vol. 32, no. 8, 2005, pp. 2013-2025.14, 15

[26] Ruben Ruiz, Funda Şerifoğlu y Thijs Urlings, "Modeling realistic hybrid flexible flowshop scheduling problems". Comput. Oper. Res., vol. 35, no. 4, 2008, pp. 1151-1175.14, 15

[27] M. Zandieh, E. Mozaffari y M. Gholami, "A robust genetic algorithm for scheduling realistic hybrid flexible flow line problems". J. Intell. Manuf., vol. 21, no. 6, 2010, pp. 731-743.14, 15, 18

[28] Mir Abbas Bozorgirad y Rasaratnam Logendran, "Hybrid flowshop scheduling problem with a bi-criteria objective and group technology assumption". in IIE Annual Conference. Proceedings, 2013, p. 3004. 14, 15, 16

[29] S. F. Attar, M. Mohammadi y R. Tavakkoli-Moghaddam, "Hybrid flexible flowshop scheduling problem with unrelated parallel machines and limited waiting times". Int. J. Adv. Manuf. Technol., vol. 68, no. 5-8, 2013, pp. 1583-1599. 14, 15

[30] Eduardo Camargo, Sergio de Souza y Marcone Jamilson,"Um algoritmo iterated greedy search aplicado à minimização do makespan”. Simpósio Brasileiro de pesquisa operacional, 2013, pp. 44-55.14, 15, 18

[31] Zhan Tao Li, Qing Chen, Ning Mao, Xiaoming Wang y Jianjun. Liu, "Scheduling rules for two-stage flexible flow shop scheduling problem subject to tail group constraint". Int. J. Prod. Econ., vol. 146, no. 2, 2013, pp. 667-678. 14

[32] Jaime Arango, Jaime Giraldo y Omar Castrillón, "Aplicando la Heurística TOC a la Secuenciación de Trabajos en un Flow Shop Híbrido Flexible". Dyna, vol. 81, no. 2011, 2014, pp. 113-119.14, 15

[33] H.-W. Kim y D.-H. Lee, "Heuristic algorithms for re-entrant hybrid flow shop scheduling with unrelated parallel 
machines". Proc. Inst. Mech. Eng. Part B J. Eng. Manuf., vol. 223, no. 4, 2009, pp. 433-442 .14

[34] Abolfazl Soltani y Behrooz Karimi, "Cyclic hybrid flow shop scheduling problem with limited buffers and machine eligibility constraints". Int. J. Adv. Manuf. Technol., vol. 76, no. 9-12, 2014, pp. 1739-1755.14, 15

[35] Omar Castrillón, Jaime Giraldo, y W. Sarache, "Técnicas inteligentes y estocásticas en scheduling”. Un enfoque en la Prod. y las operaciones. Unibiblios, Bogotá, D. C., 2010.14, 15

[36] Ruben Ruiz y Concepción Maroto, "A genetic algorithm for hybrid flowshops with sequence dependent setup times and machine eli gibility". Eur. J. Oper. Res., vol. 169, no. 3, 2006, pp. 781-800.15

[37] Pedro Gómez, "Un nuevo algoritmo genético basado en un sistema multiagente para la programación de la producción en un taller de flujo híbrido". Internacional Conference on Industrial Engineering \& Industrial Management, 2007, pp. 1675-1685.15

[38] E. Rashidi, M. Jahandar y M. Zandieh, "An improved hybrid multi-objective parallel genetic algorithm for hybrid flow shop scheduling with unrelated parallel machines". Int. J. Adv. Manuf. Technol., vol. 49, no. 9-12, 2010, pp. 1129-1139. 15

[39] Fantahun Defersha y Mingyuan Chen, "Mathematical model and parallel genetic algorithm for hybrid flexible flowshop lot streaming problem". Int. J. Adv. Manuf. Technol., vol. 62, no. 1-4, 2012, pp. 249-265. 15

[40] Pedro Gómez, Carlos Andrés y Francisco Lario, "An agent-based genetic algorithm for hybrid flowshops with sequence dependent setup times to minimise makespan". Expert Syst. Appl., vol. 39, no. 9, 2012, pp. 8095-8107. 15

[41] Pedro Luis Miranda y Rodolfo Florence Teixeira Jr, "Algoritmo genético com busca local para a programação da produção em sistemas flow- shop híbridos". Congreso Latino-iberoamericano de Investigación Operativa, 2012, pp. 19-29. 15,18

[42] Pedro Luis Miranda, Karim Martínez y Rodolfo Florence Teixeira Jr, "Um modelo de programação inteira mista para a programação da produção em flowshop híbrido com buffers limitados"., Simpósio Brasileiro de Pesquisa Operacional, 2013, pp. 154-165.15

[43] M. Jenabi, S. M. T. Fatemi Ghomi, S. a. Torabi, y B. Karimi, "Two hybrid meta-heuristics for the finite horizon ELSP in flexible flow lines with 315

[44] Ye Xu, Ling Wang, Gang Zhou y Shengyao Wang, "An effective shuffled frog leaping algorithm for solving hybrid flow-shop scheduling problem". Lect. Notes Comput. Sci. (including Subser. Lect. Notes Artif. Intell. Lect. Notes Bioinformatics), vol. 6838 LNCS, 2011, pp. 560-567.15, 18

[45] Jun-qing. Li y Quan-ke Pan, "Solving the large-scale hybrid flow shop scheduling problem with limited buffers by a hybrid artificial bee colony". 15,18

[46] B. Scholz-Reiter, H. Rekersbrink, and M. Görges, "Dynamic flexible flow shop problems - Scheduling heuristics vs. autonomous control". CIRP Ann. - Manuf. Technol., vol. 59, no. 1, 2010, pp. 465-468. 15

[47] Javier Parra Peña y Gónzalo Mejia, "Desarrollo de un modelo para la secuenciación de trabajos en la mediana industria ladrillera de la localidad XIX”, ”Tesis M.S.”, Unidandes, Colombia, 2006.

[48] Min Dai, Dunbing Tang, Adriana Giret, M. Salido, y W. D. Li, "Energy-efficient scheduling for a flexible flow shop using an improved genetic- simulated annealing algorithm". Robot. Comput. Integr. Manuf., vol. 29, no. 5, 2013, pp. 418-429. 16

[49] Catarina Dudas, Marcus Frantzén y Amos HC. NG., "A synergy of multi-objetive optimization and data mining for analysis of a flexible flow shop". Robotics and Computer-Integrated Manufacturing, vol. 27, no 4, 2011, pp. $687-695.15,16$

[50] Ruben Ruiz y Concepción Maroto, "Evaluación de Heurísticas para el Problema del Taller de Flujo". En 27 Congreso Nacional de Estadística e Investigación Operativa, 2003, pp. 1-18.17

[51] Orlando Suarez, "Una aproximación a la heuristica y metaheuristicas". INGE@ UAN, Tendencias en la Ing., vol. 1, no. 2, 2013, pp. 44-51.16

[52] Jose Francisco Chicano "Metaheurísticas e ingeniería del software". Tesis Doctoral, Lenguajes y Ciencias de la Computación, Universidad de Málaga, Málaga, España, 2007.16

[53] Sungbum Jun y Jinwoo Park, "A hybrid genetic algorithm for the hybrid flow shop scheduling problem with nighttime work and si multaneous work constraints: A case study from the transformer industry". Expert Syst. Appl., vol. 42, no. 15, 2015, pp. 6196-6204.18

[54] Sahar Safaei, Reihane Naderi, Amir Sohrabi y Amin Hatami, "Scheduling of Unrelated Parallel Machines using Two Multi Objective Genetic Algorithms with Sequence-Dependent Setup Times and Precedent Constraints". International Journal of Advanced Design and Manufacturing Technology, vol. 8, no 4, 2016. [En línea]. Disponible en: http://admt.iaumajlesi.ac.ir/index/index.php/me/article/view/983 15, 19 


\section{Eliana Marcela Peña Tibaduiza}

Ingeniera Industrial, Universidad Industrial de Santander; magíster en Ingeniería Industrial, Universidad Industrial de Santander; se desempeñó como docente tiempo completo en la Universidad Pontifica Bolivariana, Bucaramanga, Colombia. Actualmente se desempeña como profesor en el área de Dirección de Operaciones de la Escuela de Estudios Industriales y Empresariales en la Universidad Industrial de Santander de Bucaramanga; pertenece como investigador al Grupo de Organización y Optimización de Sistemas Productivos, Administrativos y Logísticos - OPALO y es coordinadora del Grupo de Aplicación de Lúdicas como Estrategia de Enseñanza-Aprendizaje -GALEA.

Correo electrónico: elmapena@uis.edu.co

\section{Edwin Alberto Garavito Hernández}

Ingeniero Industrial Universidad Industrial de Santander; especialización en Docencia Universitaria y en Gerencia de la Producción y Mejoramiento continuo, Universidad Industrial de Santander; magíster (C) en Ingeniería Industrial, Universidad de Puerto Rico; actualmente se desempeña como profesor en las áreas de Simulación y Diseño de Sistemas Productivos de la Escuela de Estudios Industriales y Empresariales en la Universidad Industrial de Santander; investigador al Grupo de Organización y Optimización de Sistemas Productivos, Administrativos y Logísticos - OPALO.

Correo electrónico: garavito@uis.edu.co

\section{Luis Eduardo Pérez Figueredo}

Ingeniero Industrial de la Universidad Industrial de Santander; profesional responsable de planeación del Instituto Municipal de Empleo y Fomento Empresarial De Bucaramanga.

Correo electrónico: lpeduardop@gmail.com

\section{Eslendis Moratto Chimenty}

Ingeniera Industrial, Universidad Industrial de Santander. Correo electrónico: esle1993@ gmail.com 